\title{
Analysis of MPLS-TP Network for Different Applications
}

\author{
N. Suhaimy ${ }^{1 *}$, W. S. H. M. W. Ahmad ${ }^{1}$, N. A M. Radzi ${ }^{1}$, F. Abdullah ${ }^{1}$, M. Z. Jamaludin ${ }^{1}$, M. N. Zakaria ${ }^{2}$ \\ ${ }^{1}$ Institute of Informatics and Computing in Energy (IICE), Electronics Communication (EC) Department, College of Engineering (COE), \\ Universiti Tenaga Nasional, 43000 Kajang, Malaysia \\ ${ }^{2}$ Architecutre and Governance, Tenaga Nasional Berhad Information and Communication Technology (TNB ICT), \\ Kuala Lumpur, Malaysia \\ *Corresponding authorE-mail: Nurshazlina@uniten.edu.my
}

\begin{abstract}
In the perspective of Multiprotocol Label Switching - Transport Profile (MPLS-TP) network, a reliable framework architecture or mechanism needs to be ascertained by comparing the results obtained through implementation of previously proposed scheme towards the network. This is important in order to implement an efficient system for different types of applications or services. Various results are reviewed and analyzed from the related works followed by detail discussions based on their proposed objectives. The analyses shown that most applications of MPLS-TP have been utilized for protection, followed by Operations, Administration and Maintenance (OAM), fairness and congestion control. This is due to the mission-critical operational service of protection which require us to meet the standard protection switching time of $50 \mathrm{~ms}$.
\end{abstract}

Keywords: Applications; Analysis; Multiprotocol Label Switching-Transport Profile (MPLS-TP); Survey.

\section{Introduction}

Multiprotocol Label Switching - Transport Profile (MPLS-TP) is a packet transport technology which is a subset and an extension of MPLS network. This technology is derived from IP-MPLS that is used to establish paths of communication by using Internet Protocol (IP) routing in order to discover network topologies [1]. However, in IP-MPLS network, these paths are unidirectional, whereas, in MPLS-TP network, these paths are guaranteed to be congruent bidirectionally $[2,3]$. This can reduce the processing delay by finding the routes only once [4].

MPLS-TP allows the deployment of MPLS in transport network with similar prediction characteristic to the existing transport networks. This technology also has the ability to operate similarly to the existing transport technologies [5].

The architecture of MPLS-TP is shown in Figure 1. Label Edge Router (LER) is operating at the edge of MPLS-TP network by determining and applying the right labels to the packets which then will be forwarded to the domain. Both ingress and egress LER routers are known as provider edge (PE) to the MPLS-TP domain. Label Switch Router (LSR) is used to switch the labels of the packets and route it through the network. Transit LSR func- tions as to switch and route the packets to the next LSR. Label Switched Path (LSP) is a route through the MPLS-TP network that been set up according to the standards in Forwarding Equivalence Class (FEC). These paths are determined by signaling protocol i.e: Label Distribution Protocol (LDP) or Border Gateway Protocol (BGP). Across the transit LSR is bidirectional LSP throughout the network.

MPLS-TP makes use of MPLS technology by applying constraint and eliminate some of the complex functions in IP-MPLS which could make the network becomes unpredictable and nondeterministic. Some of the functions that have been eliminated are Penultimate Hop Popping (PHP), Equal Cost Multi Path (ECMP) and LSP merge [6]. However, some fundamental functions of MPLS are maintained, namely the Pseudo Wire Emulation (PWE) architecture, label forwarding and dynamic control plane. Additional constraints have also been defined by the MPLS-TP on the use of MPLS in communication environment which consists of determinism, static provisioning of congruent paths, and Operations, Administration and Maintenance (OAM) functions. The characteristics of MPLS-TP network can be illustrated in Figure 2.

Transit LSR

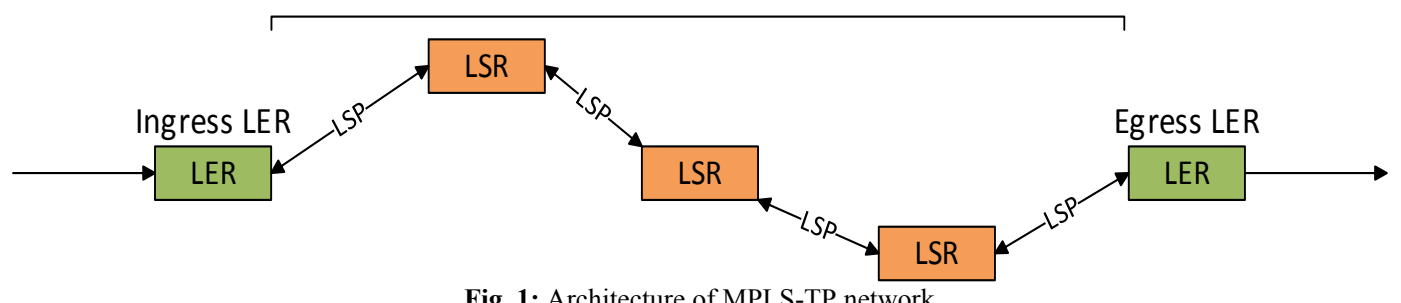

Fig. 1: Architecture of MPLS-TP network 


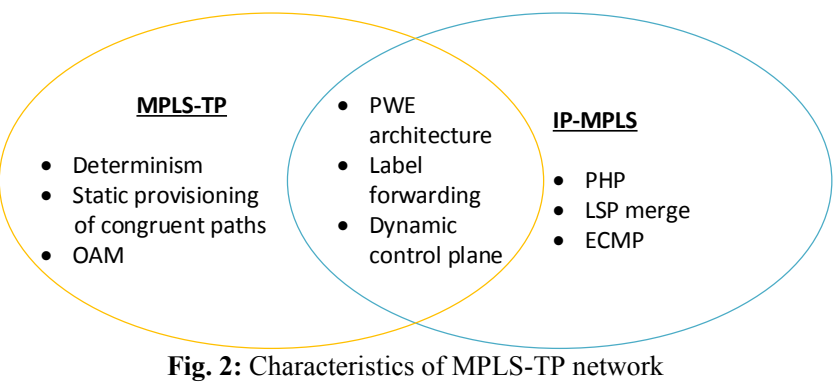

MPLS-TP can be implemented for the applications located at the edge or core network; metro or aggregation network; and also at the access network [7]. The environment of core network has high density of data traffic with limited number of sites, whereas; at the aggregation network, the traffic flow is much simpler but also critical at the same time, with high number of sites over medium size bandwidth. At the access network, there is high number of sites with diversity of connection options where the security and power will be carried out over the Ethernet. Other than that, MPLS-TP provides carrier-grade transport platform and has the predictability and resiliency of circuit-based transport network [8]. There is limited work found on the MPLS-TP network applications due to its new technology and only best fit for packet-based mission-critical operational networks. To the best of our knowledge, no analysis has yet been done on the applications of MPLS-TP network. Thus, this paper will identify and discuss different types of applications that have been proposed in the literature, which utilizing the MPLS-TP technique in packet transport services.

The rest of this paper is organized as follows. Section 2 provides an overview of the related works on MPLS-TP network applications. Section 3 explain detail methods of the related applications. Analyses and discussions of each method are presented in Section 4 and finally Section 5 provides the conclusion.

\section{Related Works}

MPLS-TP is a product from the collaboration between International Telecommunication Standardization Sector (ITU-T) and Internet Engineering Task Force (IETF) together with the industry in developing this new standard [9]. MPLS-TP offers pseudowire (PW) infrastructure, label forwarding, dynamic control plane (for fairness), determinism (for protection), static provisioning of congruent paths (congestion control) and also OAM [10], as illustrated in Figure 2. From these MPLS-TP characteristics, many applications are able to benefit from the deployment of this technology. Determinism criteria in MPLS-TP network is developed by using deterministic method, where the produced output is always the same regardless of the input. This can also be described as protection, proposed by several researchers in [11-15]. Kim et al. [11] suggested a new MPLS-TP linear protection mechanism which is known as an Optical Carrier Ethernet Switch (OCES) by using the existing Protection State Coordination (PSC) format. Ryoo et al. [12] described that linear protection switching is an important mechanism in delivering a reliable services of circuit-switched transport network especially in the event of network failure. Cortés-Polo et al. [13] introduced an Integrated Proxy Mobile MPLS-TP (IPM-TP) in providing the quality of service (QoS) in wireless heterogeneous networks. Another protection-related work by Kim et al. [14] in 2016 proposed Collective Signal Fail (C-SF) mechanism for point-to-multipoint connections in packet transport network in order to obtain fast traffic recovery. The last work found on protection application is Choi et al. [15] using Path Computation Element (PCE) based on unified control and management framework architecture for the MPLS-TP network.

In OAM, there are four main functions of it, consist of continuity check $(\mathrm{CC})$, connectivity verification $(\mathrm{CV})$, failure indication and performance monitoring [9]. Previously mentioned work by Kim et al. in [11] was also implemented for OAM. Pijanka et al. [16] presented a solution of conceptual mobile MPLS-TP with the use of OAM channels along with its advantages to optimize the handoff or handover management procedure in hierarchical network topology.

Dynamic control plane is to make deployment of MPLS-TP network operationally more efficient. This characteristic can also be defined as fairness in a network. Sakamoto et al. [17] proposed a dynamic policer control to resolve the fairness issue among services towards implementation of the integrated networks which is a transport network architecture that utilized the packet transport technology.

Congestion control in MPLS-TP is also known as static provisioning of congruent paths. Static provisioning is being used to set up static LSPs that are congruent in which that have predefined backup paths. One of the related work was proposed by Rodríguez-Pérez et al. [18], using Gossip-based Local Recovery Policy (GLRP) as a congestion control mechanism in order to improve the reliability and performance of prioritized QoS-aware services.

PW infrastructure consists of header that is used to carry time-tolive (TTL) as patented by Boutros et al. [19]. When the value of TTL is 1 , device at provider edge will be forced to undergo switching process. Whereas, when the value of TTL is greater than 1 , device will be operated in normal behavior by delivering the packet to the next provider edge without any additional process. According to Boutros et al. [19], label forwarding takes place in LSP through the initiation of CV message by the maintenance end point (MEP). Each of the CV message consists of TTL with the value of 1 for each packet. Along the LSP, number of maintenance intermediate point (MIP) receives the packet and decrements the TTL until it becomes 0. Payload field of the packet will be examined in order to perform a specific OAM function for performance measurement.

The applications of MPLS-TP network technology are summarized and presented in Table 1 .

\section{MPLS-TP Applications}

MPLS-TP is a subset of MPLS technology with a set of new functions. With the existence of new kinds of applications and technologies such as server virtualization, big data technology and data intensive applications, these have led to a higher network resources utilization which resulting in increased of bandwidth consumption and congestions in the network. This section will explain in depth on the methods of related works in MPLS-TP applications.

Kim et al. [11] proposed a new MPLS-TP linear protection mechanism which is known as an OCES by using the existing PSC format. From the perspective of network operational, it has fully satisfied the requirements of network operator, to behave like the traditional Automatic Protection Switching (APS) format. The results show that the performance of OCES as protection switching mechanism is below $50 \mathrm{~ms}$, which met the industry requirement in case of link failure. Meaning that, this solution is able to protect LSP paths since that OCES has the requirements as service providers of MPLS-TP, i.e: the availability, deterministic and scalability of packet transport network.

Linear protection switching is an important mechanism in delivering a reliable services of circuit-switched transport network especially in the event of network failure. The development of MPLSTP open standard in linear protection allows various equipment to participate with high possibility of interoperating extraordinarily since that it has been developed by both IETF, which is the home of MPLS and ITU-T, also the home of transport network. In addition, this mechanism can be implemented regardless of network topologies, either in ring or shared mesh protection switching. 
Table 1: Summary of Related Works on MPLS-TP Network

\begin{tabular}{|c|c|c|}
\hline Applications & Year & Related Works \\
\hline $\begin{array}{ll}- & \text { OAM } \\
-\quad & \text { Protection } \\
\end{array}$ & 2014 & OAM and protection mechanisms for MPLS-TP packet transport networks [11]. \\
\hline - $\quad$ Protection & 2014 & MPLS-TP linear protection for ITU-T and IETF [12]. \\
\hline - $\quad$ Protection & 2015 & $\begin{array}{l}\text { Proposal and analysis of integrated PTN architecture in the mobile backhaul to improve the QoS of } \\
\text { HetNets [13]. }\end{array}$ \\
\hline - Protection & 2016 & $\begin{array}{l}\text { Protection Switching Methods for Point-to-Multipoint Connections in Packet Transport Networks } \\
{[14] .}\end{array}$ \\
\hline - $\quad \mathrm{OAM}$ & 2016 & Mobile MPLS-TP-Support the mobility of terminal devices using OAM channel [16]. \\
\hline $\begin{array}{ll} & \text { Fairness } \\
- & \text { Bandwidth allocation } \\
\end{array}$ & 2016 & $\begin{array}{l}\text { Bandwidth Control Using Adaptive Packet Policer Management for Packet Transport Network } \\
\text { Based on Service Layer Request [17]. }\end{array}$ \\
\hline $\begin{array}{ll} & \text { Congestion control } \\
- & \text { Self-management capable } \\
& \text { routes } \\
\end{array}$ & 2015 & $\begin{array}{l}\text { An OAM function to improve the packet loss in MPLS-TP domains for prioritized QoS-aware } \\
\text { services [18]. }\end{array}$ \\
\hline $\begin{array}{ll}- & \text { PW architecture } \\
- & \text { Label forwarding } \\
\end{array}$ & 2013 & Connection verification for MPLS label switched paths and pseudowires [1]. \\
\hline
\end{tabular}

PSC and APS are pre-existing solutions for the MPLS-TP linear protection with some deficiencies, thus, an Automatic Protection Coordination (APC) has been introduced by Ryoo et al. [12] as a unified solution which could improve both of the PSC and APS solutions.

Cortés-Polo et al. [13] proposed IPM-TP which is a new architecture in providing the quality of service in wireless heterogeneous networks. The functions are to reduce the signaling cost and also to improve the QoS in heterogeneous networks with high rates of mobility. Besides, this architecture serves as the path-protection mechanism and it supports dynamic topology changes and network optimization that have been produced by the movement of the mobile nodes in heterogeneous networks. Mobility protocols together with MPLS-based packet transport network (PTN) had also been studied in order to provide the QoS in wireless heterogeneous networks.

Several protection schemes for point-to-multipoint connections in packet transport network was suggested by Kim et al. [14], in order to obtain fast traffic recovery while reusing the existing technologies of protection switching with minimal modifications. $\mathrm{C}-\mathrm{SF}$ mechanism has been introduced as a solution for per-leaf protection scheme which is related to Inter-Processor Communications (IPC), which is the time that could affect the restoration time for signal fail notifications. Tree protection scheme has been considered in order to increase the agility of this connection where a combination of tree and per-leaf protection schemes has been proposed in order to increase the network availability with a foundation of sub-50 ms. Sub-50 $\mathrm{ms}$ is the standard time constraint for protection switching as stated in $[2,5,7-9,11,12,14-16,20]$.

Choi et al. [15] proposed PCE based on unified control and management framework architecture for the MPLS-TP network. The functions are to collect the information on network topology, setting up the LSP tunnel and monitoring the states both from and to the management framework in a unified way. This architecture represents the generalized topology discovery, operational monitoring, and provisioning (G-TOP) controller by integrating the network topology discovery, path computation and also provisioning functions for carrier-grade transport network.

A solution of conceptual mobile MPLS-TP was presented by $\mathrm{Pi}$ janka et al. [16] with the use of OAM channels along with its advantages. This solution is specifically proposed for both of the aggregation and access networks by supporting the mobility of users and also to optimize the handoff or handover management procedure in hierarchical network topology. This solution can be implemented without the need to modify the current MPLS standard significantly since all signaling messages are transmitted by the standardized OAM channels.

The requirements for integrated networks was discussed by Sakamoto et al. [17] to fit in with numerous services which were previously handled by separate networks. A dynamic policer control, which is a transport network architecture that utilized the packet transport technology, has been proposed to resolve the fairness issue among services towards implementation of the integrated networks. It has been carried out through controlling bandwidth allocation by monitoring the throughputs of each services and feeding the results back to the dynamic policer controller.

Rodríguez-Pérez et al. [18] proposed GLRP as a congestion control mechanism in order to improve the reliability and performance of prioritized QoS-aware services by minimizing the amount of packet loss. GLRP also cooperates with a minimum set of extensions to resource reservation protocol traffic engineering (RSVP-TE) as self-management capable routes over MPLS-TP technology in obtaining local retransmission of lost traffic when an LSP failure occurs, in conjunction with the fast reroute pointto-point technique.

Section 4 will discuss each of the said methods and their results' behavior will be analyzed accordingly.

\section{Analyses and Discussions}

Section 3 has explained on how each method works for different MPLS-TP applications. This section will analyze and discuss their results in detail. An overall summary of the analyses will be provided at the end of this section.

To determine the performance of OCES in [11], continuity check (CC) for OAM function had been done over the LSP routes. The protection test has been carried out with an intervals of $3.3 \mathrm{~ms}$ of $\mathrm{CC}$ by imitating the customer traffic. The average of the obtained outage time is less than $16 \mathrm{~ms}$. Traffic restoration time is the time between network impairment until restoration of the traffic. Confirmation time is the time from the moment of network impairment until signal fail is triggered which produced an output between $6.7 \mathrm{~ms}$ to $9.9 \mathrm{~ms}$. Transfer time is the time interval between the triggered signal fail until the accomplishment of protection switching operations which produced an output in less than $9.3 \mathrm{~ms}$ Based on the obtained results in [11], we can relate that no loss of packets happened during the restoration process due to the merging selector, even when traffic has been rerouted from faulty path to the working path.

The compatibility and applicability of MPLS-TP in [12] can be decided using a unified linear protection switching mechanism which is APC. It has been introduced in conjunction with the preexisting solutions as well as the other topologies of network. Principles in designing the APC include:

- maintain operational behaviors of conventional network

- define auxiliary mechanisms that lacking in other transport networks

- introduce an efficient method in providing protection against signal degradation

- reuse the basic principles of operation and Protocol Data Unit (PDU) structure in PSC 
- strictly partition priority evaluation from state machine

- reduce any possible bugs in tables of state transition

Based on the obtained results in [12], we can relate that this developing unified solution is proven to have backward compatibility with the installed equipment and it is applicable to other topologies i.e: ring and shared mesh protection switching.

To determine the performance of IPM-TP in [13], the results show that, when signaling cost serves as a function of velocity of mobile node, the signaling cost of IPM-TP is lower than both of Proxy Mobile IPv6 (PMIP-MPLS) and Proxy Mobile IPv6 (PMIPv6). It is due to low number of signaling messages in order to inform the handover of mobile node and also less registered frequency that could affect the handover latency. When the packet delivery cost serves as a function of session arrival rate to a mobile node, IPMTP has lower cost than both of PMIP-MPLS and PMIPv6 due to small amount of packet loss during the handoff process. The amount of packet loss in PMIP-MPLS and PMIPv6 are higher due to the packet retransmission. When total cost is in function with the session arrival rate to mobility rate, IPM-TP has lower total cost compared to PMIP-MPLS and PMIPv6 due to the decreasing of packet delivery cost and signaling cost when mobile node is decreasing. When service blocking probability is in function with the session arrival rate to mobility rate, IPM-TP architecture allows lower probability of blocking the service due to the tunnel extension mechanism during the handover process.

The IPM-TP scheme as well as PMIP-MPLS and PMIPv6 schemes have been evaluated through signaling cost, packet delivery cost, total signaling cost and service blocking probability. The results in [13] explained that when control plane introduced a large amount of signaling messages, it will incurred a high signaling cost in mobility management protocol. This is caused by the frequent signaling or during handover process which requires tracking of the mobile nodes. The high number of messages will affect the latency of handover, which is the time when the handoff event is located until the first packet is accepted from new subnet. The approach of IPM-TP scheme could avoid packet loss by triggering the movement of signals and reroute the lost packet to the new gateway. The forwarding plane will introduce overhead if packet delivery is using tunneling mechanism, where packet delivery of mobility management protocol becomes costly. Based on the obtained results in [13], we can relate that IPM-TP can reduce the overhead by using MPLS-TP tunneling mechanism. The tota cost is presented as a function of the ratio of session arrival rateto-mobility rate. High value of ratio of session arrival rate-tomobility rate represents low mobility which incurred low cost of signaling update. Also, IPM-TP scheme can reduce the signaling and creation of new LSP tunnels in the event of handoff. This is because, the creation of new tunnels could increase the probability of blocking the service in local mobility anchor.

The C-SF mechanism in [14] has been utilized to obtain the restoration time and linear protection process (LPP) task in three different durations that serves as a function of the number of LPPs in root node. Per-leaf protection performance has been done in two conditions i.e: with and without C-SF mechanism. Based on derived equations, sum of both confirmation and transfer time serves as a function of the number of linear protection processes have matched with the experimental results. The restoration time for four different protection schemes i.e: per-leaf, ring, tree and hybrid in point-to-multipoint connection network, serves as a function of the number of affected leaf nodes. These results have been compared in bidirectional and unidirectional of signal fail.

As the number of signal fail notifications processed by the LPP task increased, the performance of protection switching has been improved. Deployment of C-SF mechanism had reduced the restoration time remarkably in order to meet the requirement of $50 \mathrm{~ms}$. Ring protection scheme has longer restoration time as compared to per-leaf protection scheme due to additional delay. As the number of affected leaf nodes increased, both hybrid and tree protection schemes are stable and not affected whereas, per-leaf and ring protection schemes are increased. Based on the obtained results in [14], we can relate that tree protection scheme has the best per- formance out of four schemes for restoration time whereas, hybrid protection scheme can improve network availability by utilizing other resources.

The feasibility of the framework in [15] is being implemented on a testbed that using MPLS-TP switches and also on G-TOP controller. From the operation testing results, it shows that the protection switching time is approximately $27 \mathrm{~ms}$, which means that the OAM framework can satisfy the strict requirements for packet transport network in carrier domain. Another results show that the performance of data forwarding for delay and throughput with different packet sizes in lossless condition recommends that the proposed protocol can be executed in actual systems with heavy traffic situations. The performance of traffic load has been carried out by using G-TOP and OpenFlow controllers whereas, the performance of the proposed protocol for LSP provisioning, path computation and link updating have been carried out by increasing the number of nodes in ring topology.

The implementation of PCE based on unified control and management framework architecture has been done by developing MPLS-TP switch which consists of data forwarding engine, OAM management engine, link layer discovery protocol management engine, and G-TOP agent engine. Whereas, for G-TOP controller it consists of controller panel, G-TOP server and traffic engineering database. The results in [15] explained that the edge nodes has to transmit a check message periodically and track the received message from the other edge nodes in a unified maintenance, in order to prove that OAM framework can satisfy the strict requirement (protection switching time of $50 \mathrm{~ms}$ ). Other than that, the proposed architecture has reduced the traffic load on G-TOP controller as compared to the amount of traffic load on OpenFlow controller. Also, the edge nodes only notify the controller in the event of failure even though the nodes periodically transmit the OAM messages. Based on the obtained results in [15], we can relate that both of LSP provisioning time and path computation time increase proportionally to the number of nodes, but not the LSP updating time. These outcomes could help in identifying the deficiency in its function and ensuring correct protocol design.

The conceptual mobile MPLS-TP with the use of OAM channels in [16], have been evaluated through an average time of transmission break during the handoff process that serves as a function of the number of edge routers (LERs) as well as the number of hierarchical network levels. Other results present number of signaling messages transmission that serves as a function of the number of edge routers (LERs) as well as the number of hierarchical network levels.

For mobile MPLS case in function of LER number, the changes in transmission break time can be seen as stepwise pattern as the number of edge router are increasing. This is due to the additional node that increased the distance between mobile node and home agent. For mobile MPLS-TP case in function of LER number, the values of transmission break time are varies in linear trend. For mobile MPLS case in function of network levels number, trend is linearly increasing due to the additional level of LSR that increased the time for signal exchange process. For mobile MPLSTP case in function of network levels number, handoff operation is done much faster due to the unnecessary to communicate with distant home agent. The number of signaling messages is related to the number of handoff process. For mobile MPLS-TP case, handoff process is more complicated than mobile MPLS. Based on the obtained results in [16], we can relate that transmission break time of mobile MPLS-TP within four levels in hierarchy is shorter than mobile MPLS. The signaling cost of mobile MPLSTP is slightly poor than time for the handoff operation.

The dynamic policer control in [17], have been evaluated through characteristics of each service traffic been affected by congestion, utilization of bandwidth serves as a function of congestion level, performance of dynamic priority control for specific application type at transport core nodes, characteristics of each service traffic serves as fair throughputs, throughputs serves as priority setting and lastly, characterize each traffic with the use of policing control 
Transmission rate of each traffic over the packet transport network depends on the link capacity at access network. Throughputs of non-priority traffic is decreasing with the developing priority traffic due to the probability of being discarded by policer control. Based on the obtained results in [17], we can relate that the transmission of packets cannot be done at maximum rate which can cause congestion on the link. Congestion on available bandwidth of each connected traffic depends on high level protocol. Policer control can be utilized in high speed networks though parallel processing technique.

In [18], performance of GLRP mechanism over various protocol parameters has been evaluated through simulation in different conditions. The effectiveness of buffer has also been measured in charts which consist of buffer-hit-ratio as a function of incoming packet ratio, meaning that the number of successful buffer reads is being divided by the total number of buffer accesses. The packet delivery ratio is analyzed in order to compare the performance and to evaluate the protocol, where the total number of delivered packets is divided by the total number of packets sent. The obtained result is a function of delivery ratio of locally recovered packets against the incoming packet ratio. The end-to-end delay as a function of packet loss is the delay for every packet delivered at the egress node.

The results in [18] explained that routers with GLRP mechanism capability had maintained one data to be buffered without considering the number of connections in serving the nodes. The larger the size of data buffer will not determine the efficiency of pro- posed policy, in fact, an optimal size of buffer is depending on the loss percentage and not on link velocity. When speed of link increases, ratio of data packets could also increase which led to lesser delivery time for local retransmission request or gossip request. Based on the obtained results in [18], we can relate that packets of data can be stored temporarily and replaced by the new incoming data packets which require optimum size of data buffer for the gossip nodes through permitting maximum diameter and congestion level in network domain. When the buffer on nodes of GLRP mechanism increases, the end-to-end delay of data packets also increases. This is due to the failure of local retransmission in forwarding data packets to the next hop caused by the congestion. By implementing GLRP mechanism, the lost packet can be found at the gossip nodes and will then be forwarded towards its destination. Other than that, the results from packet delivery ratio proved that the delivery of data packets can be improved further with different incoming buffer size and packet ratio for the network. When size of data buffer increases, the packet delivery shows no significant increase whereas, when size of data buffer decreases, the packet delivery shows a significant decrease which requires high number of end-to-end retransmission.

As can be summarized in Table 2, most applications of MPLS-TP have utilized protection mechanism, followed by OAM, fairness and congestion control. This is due to the mission-critical operational service of protection which require us to meet the standard protection switching time of $50 \mathrm{~ms}$.

\begin{tabular}{|c|c|c|c|}
\hline Authors & Methods & Results & Analysis \\
\hline Kim et al. [11]. & $\begin{array}{l}\text { Propose Optical Carrier } \\
\text { Ethernet Switch (OCES). } \\
\text { Using the existing PSC format }\end{array}$ & $\begin{array}{l}\text { Average out-of-service time of protection } \\
\text { test }<16 \mathrm{~ms} \\
6.7 \mathrm{~ms}<\text { Confirmation time }<9.9 \mathrm{~ms} \\
\text { Transfer time }<9.3 \mathrm{~ms} \\
\text { Packet loss and service interruption: at } 3.3 \\
m s \text { intervals of CC }\end{array}$ & $\begin{array}{l}\text { No packet loss during the restoration process } \\
\text { Due to merging selector } \\
\text { Traffic been rerouted from faulty path to } \\
\text { working path }\end{array}$ \\
\hline Ryoo et al. [12]. & $\begin{array}{l}\text { Introduced Automatic } \\
\text { Protection Coordination (APC) }\end{array}$ & $\begin{array}{l}\text { Maintain operational behaviours of } \\
\text { conventional network } \\
\text { Define auxiliary mechanisms lacking in } \\
\text { other transport networks } \\
\text { Introduced efficient method in providing } \\
\text { protection against signal degradation } \\
\text { Reusing basic principles of operation and } \\
\text { PDU structure in PSC } \\
\text { Strictly partitioning priority evaluation from } \\
\text { state machine } \\
\text { Reduce any possible bugs in tables of state } \\
\text { transition }\end{array}$ & $\begin{array}{l}\text { Have backward compatibility with the installed } \\
\text { equipment } \\
\text { Applicable to other topologies i.e: ring and } \\
\text { shared mesh protection switching }\end{array}$ \\
\hline $\begin{array}{l}\text { Cortés-Polo et al. } \\
\text { [13]. }\end{array}$ & $\begin{array}{l}\text { Proposed Integrated Proxy } \\
\text { Mobile MPLS-TP (IPM-TP) }\end{array}$ & $\begin{array}{l}\text { Signaling cost versus velocity of mobile } \\
\text { node } \\
\text { Packet delivery cost versus session arrival } \\
\text { rate to a mobile node } \\
\text { Total cost versus session arrival rate-to- } \\
\text { mobility rate } \\
\text { Service blocking probability versus session } \\
\text { arrival rate-to-mobility rate }\end{array}$ & $\begin{array}{l}\text { IPM-TP can reduce overhead by using MPLS- } \\
\text { TP tunneling mechanism } \\
\text { High value of ratio of session arrival rate-to- } \\
\text { mobility rate represents low mobility which } \\
\text { incurred low cost of signaling update } \\
\text { IPM-TP scheme can reduce signaling and } \\
\text { creation of new LSP tunnels in the event of } \\
\text { handoff } \\
\text { Creation of new tunnels could increase the } \\
\text { probability of blocking the service in local } \\
\text { mobility anchor }\end{array}$ \\
\hline Kim et al. [14]. & $\begin{array}{l}\text { Proposed Collective signal fail } \\
\text { (C-SF) mechanism }\end{array}$ & $\begin{array}{l}\text { Restoration time in three different durations } \\
\text { versus number of linear protection process- } \\
\text { es (for LPP task and C-SF) } \\
\text { Sum of confirmation and transfer time } \\
\text { versus number of linear protection process- } \\
\text { es } \\
\text { Restoration time versus number of affected } \\
\text { leaf nodes (bidirectional and unidirectional } \\
\text { signal fail) } \\
\text { Received data rate versus simulation time } \\
\text { Average received data rate at each link } \\
\text { versus simulation time }\end{array}$ & $\begin{array}{l}\text { Tree protection scheme has the best perfor- } \\
\text { mance out of four schemes for restoration time } \\
\text { Hybrid protection scheme can improve network } \\
\text { availability by utilizing other resources }\end{array}$ \\
\hline Choi et al. [15]. & $\begin{array}{l}\text { Proposed Path Computation } \\
\text { Element (PCE) } \\
\text { Integrates with MPLS-TP } \\
\text { switches and G-TOP controller }\end{array}$ & $\begin{array}{l}\text { Protection switching time } \approx 27 \mathrm{~ms} \\
\text { Performance of data forwarding for delay } \\
\text { and throughput with different packet sizes } \\
\text { in lossless condition }\end{array}$ & $\begin{array}{l}\text { LSP provisioning time and path computation } \\
\text { time increase proportionally to the number of } \\
\text { nodes } \\
\text { But not LSP updating time }\end{array}$ \\
\hline
\end{tabular}




\begin{tabular}{|c|c|c|c|}
\hline & & $\begin{array}{l}\text { Traffic load perform using G-TOP and } \\
\text { OpenFlow controllers } \\
\text { Proposed protocol perform by increasing } \\
\text { the number of nodes in ring topology }\end{array}$ & $\begin{array}{l}\text { Identify deficiency in its function and ensuring } \\
\text { correct protocol design }\end{array}$ \\
\hline $\begin{array}{l}\text { Sakamoto et al. } \\
{[17] .}\end{array}$ & $\begin{array}{l}\text { Propose a dynamic policer } \\
\text { control }\end{array}$ & $\begin{array}{l}\text { Traffic characteristics upon congestion } \\
\text { Bandwidth utilization versus congestion } \\
\text { level } \\
\text { Performance of dynamic priority control for } \\
\text { specific application type at transport core } \\
\text { nodes } \\
\text { Traffic characteristics in case of fair } \\
\text { throughputs } \\
\text { Throughputs vary with priority setting } \\
\text { Traffic characteristics with policing control }\end{array}$ & $\begin{array}{l}\text { Transmission of packets cannot be done at } \\
\text { maximum rate } \\
\text { Cause congestion on the link } \\
\text { Congestion on available bandwidth of each } \\
\text { connected traffic depends on high level protocol } \\
\text { Policer control can be utilized in high speed } \\
\text { networks though parallel processing technique }\end{array}$ \\
\hline $\begin{array}{l}\text { Rodríguez-Pérez et } \\
\text { al. [18]. }\end{array}$ & $\begin{array}{l}\text { Proposed Gossip-based Local } \\
\text { Recovery Policy (GLRP) } \\
\text { Cooperates with resource } \\
\text { reservation protocol traffic } \\
\text { engineering (RSVP-TE) }\end{array}$ & $\begin{array}{l}\text { Buffer-hit-ratio versus incoming packet } \\
\text { ratio } \\
\text { Delivery ratio of locally recovered packets } \\
\text { versus incoming packet ratio } \\
\text { End-to-end delay versus packet loss }\end{array}$ & $\begin{array}{l}\text { Packets of data can be stored temporarily and } \\
\text { replaced by the new incoming data packets } \\
\text { Require optimum size of data buffer for the } \\
\text { gossip nodes through permitting maximum } \\
\text { diameter and congestion level in network do- } \\
\text { main }\end{array}$ \\
\hline
\end{tabular}

\section{Conclusion}

Related works on MPLS-TP network have been briefly summarized together with the applications and services. The analysis of MPLS-TP applications has also been carried out by investigating and discussing the obtained results from several related works in providing new insights to the MPLS-TP network, which will significantly contributes to the whole community for the development of this fascinating and promising research area. Based on the analyses, we can conclude that most applications of MPLS-TP have been utilized on protection. This is due to the mission-critical operational service of protection which require us to meet the standard protection switching time of $50 \mathrm{~ms}$. However, there is limited work found on other MPLS-TP applications such as OAM, fairness, congestion control, label forwarding and PWE infrastructure. Hence, more research are suggested to be published in these mentioned area.

\section{Acknowledgement}

This research work is based on the support of TNB R\&D Fund (Seeding), U-TI-RD-18-07.

\section{References}

[1] N. M. Din, H. Hakimie, and N. Fisal, "Bandwidth sharing scheme in DiffServ-aware MPLS networks", 2007 IEEE International Conference on Telecommunications and Malaysia International Conference on Communications, (2007), pp:782-787, http://dx.doi.org/10.1109/ICTMICC.2007.4448589.

[2] S. M. Blair, C. D. Booth, J. Michielsen, and N. Joshi, "Application of MPLS-TP for transporting power system protection data", 2016 IEEE International Conference on Smart Grid Communications (SmartGridComm), (2016), http://dx.doi.org/10.1109/SmartGridComm.2016.7778830.

[3] X. Jin, A. Wen, W. Huang, N. Liu, C. Wei, J. Zhang, and Z. Ren, "An efficient algorithm for rapidly establishing bidirectional label switch paths based on single trips of control packets", Dianzi $Y u$ Xinxi Xuebao/Journal of Electronics and Information Technology, Vol.38, (2016), pp:707-712, http://dx.doi.org/10.11999/JEIT150754.

[4] N. A. M. Radzi, M. A. Ridwan, F. Abdullah, N. M. Din, M. H. AlMansoori, and H. Z. Abidin, "Effects of varying fiber length towards packet delay in Passive Optical Network", 2016 IEEE Region 10 Conference (TENCON), (2016), pp:3635-3638, http://dx.doi.org/10.1109/TENCON.2016.7848735.
[5] J. Rak, M. Pickavet, K. S. Trivedi, J. A. Lopez, A. M. Koster, J. P. Sterbenz, E. K. Çetinkaya, T. Gomes, M. Gunkel, and K. Walkowiak, "Future research directions in design of reliable communication systems", Telecommunication Systems, Vol.60, (2015), pp:423-450, http://dx.doi.org/10.1007/s11235-015-9987-7.

[6] M. Murakami and Y. Koike, "Highly Reliable and Large-Capacity Packet Transport Networks: Technologies, Perspectives, and Standardization", Journal of Lightwave Technology, Vol.32, (2014), pp:805-816, http://dx.doi.org/10.1109/JLT.2013.2293510.

[7] N. Haddaji, K. Nguyen, and M. Cheriet, "Towards end-to-end integrated optical packet network: Empirical analysis", Optical Switching and Networking, Vol.27, (2018), pp:18-39, http://dx.doi.org/10.1016/j.osn.2017.06.003.

[8] S. Mehta, S. Joshi, T. Das, and A. Gumaste, "On control plane algorithms for Carrier Ethernet networks: Unicast, multicast provisioning and control traffic reduction", Optical Switching and Networking, Vol.23, (2017), pp:52-66, https://doi.org/10.1016/j.osn.2016.09.001.

[9] D. M. Sul, S. H. Byun, T. I. Kim, and J. H. Lee, "A return path bandwidth management method for point to multipoint in MPLSTP", 2014 International Conference on Information and Communication Technology Convergence (ICTC), (2014), pp:794797, http://dx.doi.org/10.1109/ICTC.2014.6983291.

[10] M. Azizi, R. Benaini, and M. B. Mamoun, "Delay measurement in OpenFlow-enabled MPLS-TP network", Modern Applied Science, Vol.9, No.3, (2015), pp:90, http://dx.doi.org/10.5539/mas.v9n3p90.

[11] D.-U. Kim, Y.-C. Ryoo, B. Kim, and J. Lee, "OAM and protection mechanisms for MPLS-TP packet transport networks", Optical Internet 2014 (COIN), 2014 12th International Conference, (2014), pp:1-4, http://dx.doi.org/10.1109/COIN.2014.6950605.

[12] J.-d. Ryoo, T. Cheung, D. King, A. Farrel, and H. van Helvoort, "MPLS-TP linear protection for ITU-T and IETF", IEEE Communications Magazine, Vol.52, (2014), pp:16-21, http://dx.doi.org/10.1109/MCOM.2014.6979981.

[13] D. Cortés-Polo, J.-L. González-Sánchez, J. Carmona-Murillo, and F. J. Rodríguez-Pérez, "Proposal and analysis of integrated PTN architecture in the mobile backhaul to improve the QoS of HetNets", EURASIP Journal on Wireless Communications and Networking, Vol.2015, (2015), pp:116, https://doi.org/10.1186/s13638-015$0341-2$.

[14] D. U. Kim, J. d. Ryoo, J. H. Lee, B. C. Kim, and J. Y. Lee, "Protection Switching Methods for Point-to-Multipoint Connections in Packet Transport Networks", Etri Journal, Vol.38, (2016), pp:18-29, http://dx.doi.org/10.4218/etrij.16.0115.0024.

[15] J. S. Choi, "Design and implementation of a stateful PCE-based unified control and management framework for carrier-grade MPLS-TP networks", Journal of Lightwave Technology, Vol.34, (2016), pp:836-844, https://doi.org/10.1109/JLT.2015.2502238.

[16] M. M. Pijanka and G. W. Różański, "Mobile MPLS-TP-Support the mobility of terminal devices using OAM channel", 
International Journal of Electronics and Telecommunications,
Vol.62,
No.3,
(2016),
pp:215-223,

http://dx.doi.org/10.1515/eletel-2016-0029.

[17] K. Sakamoto, H. Hoshihara, H. Kubo, and T. Takahashi, "Bandwidth Control Using Adaptive Packet Policer Management for Packet Transport Network Based on Service Layer Request", Electronics and Communications in Japan, Vol.99, No.8, (2016), pp:38-47, https://doi.org/10.1002/ecj.11837.

[18] F. J. Rodríguez-Pérez, J. L. González-Sánchez, J. Carmona-Murill o, and D. Cortés-Polo, "An OAM function to improve the packet loss in MPLS-TP domains for prioritized QoS- aware services", International Journal of Communication Systems, Vol.28, (2015), pp:1037-1052, https://doi.org/10.1002/dac.2742.

[19] S. Boutros, M. Sivabalan, D. D. Ward, G. Swallow, S. Bryant, and I. M. C. Shand, "Connection verification for MPLS label switched paths and pseudowires", US20100238788A1, US Patent, (2013).

[20] J. S. Choi, "Design and implementation of a PCE-based softwaredefined provisioning framework for carrier-grade MPLS-TP networks," Photonic Network Communications, Vol.29, (2015), pp:96-105, https://doi.org/10.1007/s11107-014-0472-0. 